\title{
\#MaskUp in Australia: How social norms in a pandemic are formed
}

Edition 6, 2021

Dr Jun Ohashi

DOI: 10.37839/MAR2652-550X6.7

Between July and November 2020, most of the residents of Australia's second most populous state, Victoria, were, for the first time, required to wear face masks at all times outside their homes. Mask-wearing in Melbourne was made mandatory by the State Government in late July with a \$200 fine for non-compliance, after a second wave of COVID-19 infections began, and other parts of Victoria followed.

Before this, the Australian public had been provided with conflicting advice on maskwearing and its effectiveness in virus control, from governments, medical experts, community leaders and media. On July 1 2020, Australia's then acting chief medical officer, Professor Paul Kelly, stated that masks are not necessary 'in most circumstances for most people, most of the time', and responded 'it is an option' to a question 'should people in hotspot areas be wearing masks, yes or no?'.

Mandatory mask-use meant the public had to adopt a new habit. The change created an intriguing space where multiple ideologies and group identities formed and met cross-sectionally. These colliding perspectives were expressed on social media and had the power to influence people's behavior through the process of norm construction.

\section{Social norms and social identity theory}

Social norms are understood here, as articulated by scholar Jon Elster, as 'emotional and behavioural propensities of individuals', and thus emergent and fluid. 
They reside in social as well as psychological domains of human activities; in other words they are 'shared by other people and partly sustained by their approval and disapproval' and also 'by the feelings of embarrassment, anxiety, guilt and shame that a person suffers at the prospect of violating them'. It is believed that both domains influence human behaviours, and thus according to scholars Katharine Steentjes, Tim Kurz, Manuela Barreto and Thomas Morton, '[i]nformation about what others think one should do (injunctive norms) and what they actually do (descriptive norms) has been shown to crucially influence individuals' decisions to think and/or behave in particular ways'.

I argue that social identities and social norms are closely related, in the sense that the symbolic meaning of mask-wearing involves people's understanding, and enacting, of social norms with which they position and categorise themselves and others. According to social identity theory scholars Henri Tajfel and John Turner, people accentuate intergroup differences. In other words, they amplify positive attributes of their perceived in-group members in relation to out-group members, who are negatively evaluated in order for the in-group members to attain positive social identity.

Online discussion forums provide us with naturally occurring interactive data. I focused on Reddit, which is the seventh most visited website in Australia (after Google, YouTube, Facebook and others). In particular, data has been drawn from a subreddit group which is specifically dedicated to 'Melburnians and Melbourne enthusiasts', which is the biggest social online forum focusing on issues in Melbourne, with 290,000 registered members.

The coverage of the data for this article is a thread called, 'I'm kinda proud of Melbourne right now' which was initially posted by RP1 (Reddit Participant 1) on July 25 2020, three days after face coverings were mandated in metropolitan Melbourne. There were 756 comments over the next 24 hours or so, attracting 2,900 positive votes. Reddit has an inbuilt voting function, which shows whether a post is interpreted positively or negatively. Underneath each post, upward (1 point) and 
downward (-1 point) arrows set. Observers can click on either of them to indicate their approval/disapproval (or just leave unclicked). Therefore, the total score given to a post is a good indication of what is interpreted positively or negatively. It can be said that posts with more votes reflect social norms of the community. Therefore 2,900 votes indicate that many participants interpreted the initial post sent by RP1 positively.

This post by RP1 describes her/his observation of Melburnians' mask-wearing behaviours a few days after mask-wearing was mandated.

'Day 3 of masks. Holy shit I'm impressed with how immediately almost everyone I've seen has gotten onboard with the face masks. So far, I've only seen a small group of high school kids just wearing the masks around their necks instead of on their faces, but everyone has a mask. (granted, I'm spending maybe 45 mins walking out of the house at the moment) [sic].'

In what follows, I identified frequently occurring vocabulary used by participants to evaluate non-mask wearing, because this vocabulary is a key indicator of participants' positioning and categorisation of themselves and others. If participants use positive evaluative labels, 'good' for instance, to describe those who wear masks, they position themselves as pro-masks. Further, if participants use words such as 'bad' to negatively evaluate those who do not wear masks, they also assert their promask identity. If more positive evaluative labels than negative ones are used to describe those who wear masks, I conclude that mask-wearing is a social norm in this community. The reverse scenario is also possible: if negative descriptors such as 'bad' are used to describe those who wear masks, participants are establishing their anti-mask identity.

Once a mask-wearing, or non-mask wearing, practice is established as a social norm, it influences the behaviour of others. I also analysed how participants responded to a particular participant's evaluation of non-mask wearing, and how the group jointly established a social identity and sense of community, or in other words, 'in- 
groupness'.

\section{Key linguistic labels used by Reddit users to evaluate mask-wearing}

In the discussions I analysed, the most frequently occurring evaluative labels were 'good'/'great' and 'bad', which occurred 39/16 and 19 times respectively.

\section{The use of the terms 'good'/'great' and 'bad'}

'Good'/'great' and 'bad' are the most simple and direct evaluative labels, the former being associated with positive evaluation of a chosen object and the latter negative. Irony may have allowed individuals to imply the opposite, however, unless other participants interpret the implication and respond to it accordingly, irony is not established. There are no 'established' examples in the data I studied.

'Good'/'great' and 'bad' are, however, not exclusively used to evaluate behaviors in relation to mask-wearing in the thread. Some talked about 'good hygiene', some used phrases such as 'good point' and 'good work' to acknowledge and praise comments and information posted by other contributors. There are a few examples that 'good' is used to evaluate mask-wearing behaviours. For example, RP2 (Reddit Participant 2) posted the comment 'Good work, Melbourne!' which was their positive evaluation of compliance with the mask-wearing mandate. Comments such as '[G]ood to see' were used in a similar context by other participants. 'Great' is used similarly to 'good' but was usually tied to positive evaluation of mask-wearing behaviours, for example, 'Victoria is doing great', 'great we wear masks', 'great to see', and 'it's been great', all of which refer to mask-wearing.

The following use of 'good' as a positive evaluative label in relation to mask-wearing contributes to forming pro-mask social identity. RP3 [Reddit Participant 3] posted 'Good on her at least she's aware of herself' in reaction to previous posts where 
participants shared anecdotes about a person who had forgotten to wear a mask and apologetically ran back to the car to get it. At the time these comments were posted, mask-wearing was a new requirement and it is suspected that there were many instances of this kind. In any case, it is interesting to observe in this sequence of posts how social actors jointly create social norms and social identity.

'Bad' is a negative evaluative label which is potentially used to claim one's position by 'othering' those who are identified as the 'out-group'. In the data studied, 'bad' is used typically to describe the consequences of the health response to the COVID-19 pandemic, including the discomfort of wearing a mask and negative economic impacts. However, interestingly 'bad' was not often used as a label to evaluate those who complied or did not comply with mandated mask-wearing. There is only one use of 'bad'-'a really bad idea'-referring to someone not wearing a mask.

\section{The use of the terms 'idiot' and 'stupid'}

Other notable negative evaluative labels include 'idiot' and 'stupid', occurring 11 and six times respectively. They are used to evaluate non-mask wearing, and therefore claiming a participant's positioning by othering or identifying out-group members. Idiot is used to refer to instances of non-conformity with mask-wearing in Melbourne and other contexts, including the then US president Donald Trump and his followers. RP4 (Reddit Participant 4] posted:

'In the last day or so ive [sic] only seen the occasional idiot who puts their own comfort above the wellbeing of the community.'

There are three cases where 'stupid' is used to describe those who do not fully comply with the mask mandate. In other cases, 'stupid' is used to express disapproval of US government policies on the COVID-19 pandemic in general. There are two counts of 'moron', and all refer to people not wearing masks.

Participants use negative evaluative labels to disapprove of those who do not comply with the mask-wearing mandate, and to claim a pro-mask identity. The more 
negative evaluations are attached to people not wearing masks, the more such negative views are consolidated as social norms within this community.

\section{The use of the terms 'proud' and 'happy'}

It appears that a participant can claim pro-mask identity by imposing negative labels to those who do not wear masks, but that alone is not enough to form a sense of community or pro-mask social identity. The following sequence of comments include 'proud' and 'happy' both of which are evaluative as well as emotive vocabulary which appear to be more effective than negative evaluative labels for the formation of social identity.

'I'm so proud of everyone! I feel like it's bringing us all closer together in some way.. helping us connect as a community' RP5 [Reddit Participant 5]

'This is why Melbourne is going to kill this sucker quickly and efficiently. So proud of my home town.' RP6 [Reddit Participant 6]

'I really do think everyone are fed up and are being compliant so we can get this over and done with. I believe perhaps we should've done this the first time around, however I'm happy to be seeing masks around as well'. RP7 [Reddit Participant 7]

The comment by RP5 follows a series of posts observing mask-wearing situations in various parts of metropolitan Melbourne. In this comment, 'proud' is used to show her/his positive evaluation, together with 'us', 'closer together', 'connect', and 'community' all of which contribute to the social identity of those who are pro-masks, and their in-group formation. RP6 appears to agree, using the word 'proud' and describing Melbourne as her/his 'home town', amplifying in-groupness. RP7 does not fully embrace the emerging social identity, but critically evaluates the lack of voluntary mask-wearing at earlier stages of the pandemic. Nevertheless, s/he positively evaluates growing numbers of 'mask people' by using the term 'happy'.

'Proud' occurs in the discussion analysed 15 times including the examples above. 
Many are used in posts reacting to mask-wearing compliance. 'Happy' occurs 12 times, but only four are related to compliance to the mask-wearing mandate. 'Happy' appears to have more general use, as in 'I'm happy to explain'. Further examples of the use of 'proud' and 'happy' are included here:

'Thank you! Such a proud day for me.' RP8 [Reddit Participant 8]

'Its good now that's there is something that we can feel proud of our city again.[sic]' RP9 [Reddit Participant 9]

'I'm proud of us as well the two times I've left the house $99 \%$ of people have been wearing masks which is great, ' RP10 [Reddit Participant 10]

'I'm so proud *most* people are trying their best during these times' RP11 [Reddit Participant 11]

'Did a grocery trip and was happy to see really good compliance overall'. RP12 [Reddit Participant 12]

'I have also been happy to see the masked faces when I'm out walking.' RP13 [Reddit Participant 13]

All the above uses of 'proud' and 'happy' positively evaluate situations where people in Melbourne complied with the mask-wearing mandate. However, there are a few posts which question those who are 'proud' of the situation-some participants felt many people would not wear masks if it wasn't mandatory. These commentators were 'pro-mask', but positioned themselves differently. The following post by RP14 [Reddit Participant 14] distances him/herself from 'being proud' but shows some understanding to those who are proud of Melburnians' mask-wearing behaviours and claims pro-mask identity.

'Lol [Laugh out loud] idk [I don't know] about being "proud" bc [because] we're not being entitled [we're] irresponsible babies when the rest of the world adopted masks ages ago, but I understand your sentiment. Reasonable behaviour by melburnians 
[sic] for once.' RP14

Some pro-mask participants were critical of the way compliance was achieved in Melbourne. Both 'proud' and 'happy' play a similar function in terms of indicating a pro-mask stance, however, being 'proud' of the collective behaviours of Melburnians was subject to counter argument and criticism. It appears that 'pride' is associated with positive evaluation and emotion in relation to collective achievement. Being 'happy' is a positive feeling about anything, but does not claim what 'being proud' would. The use of 'proud' has stronger association with in-group formation, therefore some pro-mask participants rejected being naively proud but being critical in order to state we could do better.

\section{Conclusion}

In the Reddit thread analysed here, the majority of participants contributed at least several posts and in so doing engaged in discursive practices of meaning-making 'here and now', positively or negatively evaluating the non-mask wearing behaviours of fellow Melburnians. As they do so, they 'other' out-group attributes (not wearing a mask) and form new social identities. These positions simultaneously reflect, inform and contribute to ever-changing social norms.

The Reddit discussion participants contribute to the creation of ever-changing social norms, which are constantly challenged, reimagined, dissolved, revived. Even simple evaluative language plays an important role in creating social norms and grouporiented social identities. The discussion forum in Reddit was a microcosm of the wider Melbourne community and appears to reflect the widespread sentiment of Melburnians in relation to mask-wearing as a public health measure.

Related webinar: Governance or social resilience: Learning from Southeast Asia's experience with COVID-19.

Image: A woman wearing a face mask in Melbourne, Australia. January 2020. Credit: 


\section{MELBOURNE ASIA REVIEW}

April 20, 2021

Alpha/Flickr. 